\title{
STATES ON PSEUDO EFFECT ALGEBRAS AND INTEGRALS
}

\author{
ANATOLIJ DVUREČENSKIJ \\ 1 Mathematical Institute, Slovak Academy of Sciences \\ Štefánikova 49, SK-814 73 Bratislava, Slovakia \\ E-mail: dvurecen@mat.savba.sk,
}

\begin{abstract}
We show that every state on an interval pseudo effect algebra $E$ satisfying some kind of the Riesz Decomposition Properties (RDP) is an integral through a regular Borel probability measure defined on the Borel $\sigma$ algebra of a Choquet simplex $K$. In particular, if $E$ satisfies the strongest type of (RDP), the representing Borel probability measure can be uniquely chosen to have its support in the set of the extreme points of $K$.
\end{abstract}

\section{INTRODUCTION}

At the beginning of the Nineties, Foulis and Bennett $[\mathrm{FoBe}$ introduced effect algebras with a partially defined addition,,+ in order to axiomatize some quantum measurements. They reflect common features of the quantum logic $\mathcal{P}(H)$ of all orthogonal projectors of a Hilbert space $H$, that is a complete orthomodular lattice, and of the set of all Hermitian operators between the operators $O$ and $I, \mathcal{E}(H)$, that models so-called POV-measures.

Effect algebras generalize many examples of quantum structures, like Boolean algebras, orthomodular lattices or posets, orthoalgebras, MV-algebras, etc. We recall that $\mathrm{MV}$-algebras are algebraic counterparts of the many-valued reasoning, and they appeared in Mathematics under many different names, situations and motivations. Even in the theory of effect algebras, they were defined in an equivalent way as Phi-symmetric effect algebras, $\mathrm{BeFo}$. The monograph $\mathrm{DvPu}$ can serve as a basic source of information about effect algebras.

Many important examples of effect algebras can be obtained as an interval in the positive cone of a partially ordered group (= po-group). For example, if $\mathcal{B}(H)$ denotes the system of all Hermitian operators, then it is a po-group with respect to the natural ordering of operators, and $\mathcal{E}(H)=[O, I] \subset \mathcal{B}(H)$.

During the last decade, a whole family of interesting structures has been appeared, like pseudo MV-algebras, GeIo, where some kind of commutativity was dropped. The author and Vetterlein introduced a non-commutative version of effect algebras, called pseudo effect algebras, see DvVe1, DvVe2. If a pseudo effect algebra satisfies a stronger version of the Riesz Decomposition Property, (RDP $)_{1}$, then it is also an interval in some not necessarily commutative po-group satisfying also

\footnotetext{
${ }^{1}$ Keywords: Pseudo effect algebra; effect algebra; Riesz Decomposition Properties; state; unital po-group; simplex; Choquet simplex; Bauer simplex

AMS classification: 81P15, 03G12, 03B50

The author thanks for the support by Center of Excellence SAS - Quantum Technologies -, ERDF OP R\&D Projects CE QUTE ITMS 26240120009 and meta-QUTE ITMS 26240120022, the grant VEGA No. 2/0032/09 SAV.
} 
$(\mathrm{RDP})_{1}$, DvVe2. In addition, there is even a categorical equivalence among such structures, the category of pseudo effect algebras with (RDP) $)_{1}$ and the category of unital po-groups with $(\mathrm{RDP})_{1}$ that are not necessarily Abelian.

A state is an analogue of a probability measure for quantum structures. It is defined as a normalized additive functional on a pseudo effect algebra preserving the partial addition + . In many cases it is connected with a state on a unital po-group.

The state space of a pseudo effect algebra is always a compact convex set, unfortunately, sometimes it is empty. Recently, Panti $[\mathrm{Pan}$ and Kroupa [Kro proved that every state on an MV-algebra (= Phi-symmetric effect algebra) can be represented as an integral through a regular Borel probability measure. This result was generalized in Dvu2 also for interval effect algebras. In this study we continue with states on pseudo effect algebras, and this is the main aim of the present paper. For this goal, we show that if a pseudo effect algebra satisfies either (RDP) $)_{1}$ or it is an interval in a unital po-group with (RDP), then its state space is either empty or it is a nonempty Choquet simplex. If $E$ is even a pseudo effect algebra with $(\mathrm{RDP})_{2}$, then its state space is either empty or a nonempty Bauer simplex. To show that it is necessary to study relatively bounded homomorphisms on nonAbelian po-groups. These notions are studied in the monograph Goo, pp. 37-42] only for Abelian po-groups. Because we are working with po-groups that are not necessarily commutative groups, it is necessary to exhibit these homomorphisms for our case in full details.

Finally, this will allow us to represent any state as a standard integral through a regular Borel probability measure over the Borel $\sigma$-algebra generated by the state space.

The paper is organized as follows. Section 2 is a review on pseudo effect algebras and their basic properties. Relatively bounded homomorphisms for not necessarily Abelian po-groups are studied in Section 3. The state spaces of pseudo effect algebras and the situations when they are simplices are studied in Section 4. The main body of the paper, the integral representation of states on pseudo effect algebras, is exhibited in Section 5. Some final remarks are presented in the last section.

\section{Pseudo Effect Algebras}

According to [DvVe1, Dvu2], a pseudo effect algebra is a partial algebra $(E ;+, 0,1)$, where + is a partial binary operation and 0 and 1 are constants, such that for all $a, b, c \in E$, the following holds

(i) $a+b$ and $(a+b)+c$ exist if and only if $b+c$ and $a+(b+c)$ exist, and in this case $(a+b)+c=a+(b+c)$;

(ii) there is exactly one $d \in E$ and exactly one $e \in E$ such that $a+d=e+a=1$;

(iii) if $a+b$ exists, there are elements $d, e \in E$ such that $a+b=d+a=b+e$;

(iv) if $1+a$ or $a+1$ exists, then $a=0$.

If we define $a \leq b$ if and only if there exists an element $c \in E$ such that $a+c=b$, then $\leq$ is a partial ordering on $E$ such that $0 \leq a \leq 1$ for any $a \in E$. It is possible to show that $a \leq b$ if and only if $b=a+c=d+a$ for some $c, d \in E$. We write $c=a / b$ and $d=b \backslash a$. Then

$$
(b \backslash a)+a=a+(a / b)=b,
$$

and we write $a^{-}=1 \backslash a$ and $a^{\sim}=a / 1$ for any $a \in E$. 
For basic properties of pseudo effect algebras see [DvVe1] and DvVe2]. We recall that if + is commutative, $E$ is said to be an effect algebra; for a comprehensive overview on effect algebras see e.g. $\mathrm{DvPu}$.

We recall that a po-group (= partially ordered group) is a group $G$ with a partial order, $\leq$, such that if $a \leq b, a, b \in G$, then $x+a+y \leq x+b+y$ for all $x, y \in G$. We denote by $G^{+}$the set of all positive elements of $G$. If, in addition, $\leq$ implies that $G$ is a lattice, we call it an $\ell$-group (= lattice ordered group). An element $u \in G^{+}$is said to a strong element if given $g \in G$, there is an integer $n \geq 1$ such that $g \leq n u$, and the couple $(G, u)$ with a fixed strong is called a unital po-group. The books like [Fuc, Gla] can serve as guides through the world of partially ordered groups.

For example, if $(G, u)$ is a unital (not necessary Abelian) po-group with strong unit $u$, and

$$
\Gamma(G, u):=\{g \in G: 0 \leq g \leq u\},
$$

then $(\Gamma(G, u) ;+, 0, u)$ is a pseudo effect algebra if we restrict the group addition + to $\Gamma(G, u)$. Every pseudo effect algebra $E$ that is isomorphic to some $\Gamma(G, u)$ is said to be an interval pseudo effect algebra.

We recall that if $a^{-}=a^{\sim}$ for all $a \in E,(E$ is said to be symmetric), then $E$ is not necessarily commutative. E.g., let $\mathbb{Z}$ be the group of integers and $G$ be a non-Abelian po-group, and let $\mathbb{Z} \overrightarrow{\times} G$ be the lexicographical product; $u=(0,1)$ is its strong unit. Then $E=\Gamma((\mathbb{Z} \overrightarrow{\times} G,(1,0))$ is a symmetric pseudo effect algebra that is not commutative.

According to DvVe1, we introduce for pseudo effect algebras the following forms of the Riesz Decomposition Properties which in the case of commutative effect algebras can coincide:

(a) For $a, b \in E$, we write $a \operatorname{com} b$ to mean that for all $a_{1} \leq a$ and $b_{1} \leq b, a_{1}$ and $b_{1}$ commute.

(b) We say that $E$ fulfils the Riesz Interpolation Property, (RIP) for short, if for any $a_{1}, a_{2}, b_{1}, b_{2} \in E$ such that $a_{1}, a_{2} \leq b_{1}, b_{2}$ there is a $c \in E$ such that $a_{1}, a_{2} \leq c \leq b_{1}, b_{2}$.

(c) We say that $E$ fulfils the weak Riesz Decomposition Property, $\left(\mathrm{RDP}_{0}\right)$ for short, if for any $a, b_{1}, b_{2} \in E$ such that $a \leq b_{1}+b_{2}$ there are $d_{1}, d_{2} \in E$ such that $d_{1} \leq b_{1}, d_{2} \leq b_{2}$ and $a=d_{1}+d_{2}$.

(d) We say that $E$ fulfils the Riesz Decomposition Property, (RDP) for short, if for any $a_{1}, a_{2}, b_{1}, b_{2} \in E$ such that $a_{1}+a_{2}=b_{1}+b_{2}$ there are $d_{1}, d_{2}, d_{3}, d_{4} \in$ $E$ such that $d_{1}+d_{2}=a_{1}, d_{3}+d_{4}=a_{2}, d_{1}+d_{3}=b_{1}, d_{2}+d_{4}=b_{2}$.

(e) We say that $E$ fulfils the commutational Riesz Decomposition Property, $\left(\mathrm{RDP}_{1}\right)$ for short, if for any $a_{1}, a_{2}, b_{1}, b_{2} \in E$ such that $a_{1}+a_{2}=b_{1}+b_{2}$ there are $d_{1}, d_{2}, d_{3}, d_{4} \in E$ such that (i) $d_{1}+d_{2}=a_{1}, d_{3}+d_{4}=a_{2}$, $d_{1}+d_{3}=b_{1}, d_{2}+d_{4}=b_{2}$, and (ii) $d_{2}$ com $d_{3}$.

(f) We say that $E$ fulfils the strong Riesz Decomposition Property, $\left(\mathrm{RDP}_{2}\right)$ for short, if for any $a_{1}, a_{2}, b_{1}, b_{2} \in E$ such that $a_{1}+a_{2}=b_{1}+b_{2}$ there are $d_{1}, d_{2}, d_{3}, d_{4} \in E$ such that (i) $d_{1}+d_{2}=a_{1}, d_{3}+d_{4}=a_{2}, d_{1}+d_{3}=b_{1}$, $d_{2}+d_{4}=b_{2}$, and (ii) $d_{2} \wedge d_{3}=0$.

We have the implications

$$
\left(\mathrm{RDP}_{2}\right) \Rightarrow\left(\mathrm{RDP}_{1}\right) \Rightarrow(\mathrm{RDP}) \Rightarrow\left(\mathrm{RDP}_{0}\right) \Rightarrow(\mathrm{RIP})
$$

The converse of any of these implications does not hold, see [DvVe1]. For commutative effect algebras we have

$$
\left(\mathrm{RDP}_{2}\right) \Rightarrow\left(\mathrm{RDP}_{1}\right) \Leftrightarrow(\mathrm{RDP}) \Leftrightarrow\left(\mathrm{RDP}_{0}\right) \Rightarrow(\mathrm{RIP}) \text {. }
$$


In addition, every pseudo effect algebra with $(\mathrm{RDP})_{2}$ is lattice-ordered, DvVe1, Prop 3.3].

We note that if $E$ is an effect algebra with (RDP), then $E$ is an interval pogroup, see $\mathrm{Rav}$ ( $(\mathrm{DvPu}$, Thm 1.7.17]), and also if $E$ is a pseudo effect algebra with $(\mathrm{RDP})_{1}$, then $E$ is an interval pseudo effect algebra, see [DvVe2, Thm 5.7].

According to [GeIo, a pseudo $M V$-algebra is an algebra $\left(M ; \oplus,^{-}, \sim, 0,1\right)$ of type $(2,1,1,0,0)$ such that the following axioms hold for all $x, y, z \in M$, where the derived operation $\odot$ appearing in the axioms (A6) and (A7) is defined by

$$
y \odot x=\left(x^{-} \oplus y^{-}\right)^{\sim} .
$$

(A1) $x \oplus(y \oplus z)=(x \oplus y) \oplus z$;

(A2) $x \oplus 0=0 \oplus x=x$;

(A3) $x \oplus 1=1 \oplus x=1$;

(A4) $1^{\sim}=0 ; 1^{-}=0$

(A5) $\left(x^{-} \oplus y^{-}\right)^{\sim}=\left(x^{\sim} \oplus y^{\sim}\right)^{-}$;

(A6) $x \oplus\left(x^{\sim} \odot y\right)=y \oplus\left(y^{\sim} \odot x\right)=\left(x \odot y^{-}\right) \oplus y=\left(y \odot x^{-}\right) \oplus x$;

(A7) $x \odot\left(x^{-} \oplus y\right)=\left(x \oplus y^{\sim}\right) \odot y$;

(A8) $\left(x^{-}\right)^{\sim}=x$.

For a unital $\ell$-group $(G, u)$, set

$$
\Gamma(G, u):=[0, u]
$$

and

$$
\begin{aligned}
x \oplus y & :=(x+y) \wedge u, \\
x^{-} & :=u-x, \\
x^{\sim} & :=-x+u, \\
x \odot y & :=(x-u+y) \vee 0,
\end{aligned}
$$

then $\left(\Gamma(G, u) ; \oplus,^{-}, \sim, 0, u\right)$ is a pseudo MV-algebra, and according to [Dvu1, for any pseudo $\mathrm{MV}$-algebra there is a unique unital $\ell$-group $(G, u)$ such that $M \cong$ $\Gamma(G, u)$.

Define + to be a partial operation on $M$ that is defined for elements $a, b \in M$ iff $a \leq b^{-}$, and in that case let $a+b:=a \oplus b$. Then $(M ;+, 0,1)$ is a pseudo effect algebra satisfying $(\mathrm{RDP})_{2}$, and conversely, every pseudo effect algebra satisfying $(\mathrm{RDP})_{2}$ can be transformed into a pseudo MV-algebra, see [DvVe2, Thm 8.8].

We say that a mapping $s$ from a pseudo effect algebra $E$ into the real interval $[0,1]$ is a state if $s(a+b)=s(a)+s(b)$ whenever $a+b$ is defined in $E$ and $s(1)=1$. Let $\mathcal{S}(E)$ be the set of all states. Then it is a convex set, i.e. if $s_{1}, s_{2} \in \mathcal{S}(E)$ and $\lambda \in[0,1]$, then $s=\lambda s_{1}+(1-\lambda) s_{2} \in \mathcal{S}(E)$. It can happen that $\mathcal{S}(E)$ is empty. A state $s$ is extremal if from the property $s=\lambda s_{1}+(1-\lambda) s_{2}$ for some $s_{1}, s_{2} \in \mathcal{S}(E)$ and $\lambda \in(0,1)$, we conclude $s=s_{1}=s_{2}$. Let $\partial_{e} \mathcal{S}(E)$ denote the set of all extremal states on $E$.

In a similar way we define also a state on any pseudo MV-algebra.

We say that a net of states, $\left\{s_{\alpha}\right\}$, on $E$ converges weakly to a state, $s$, on $E$ if $\lim _{\alpha} s_{\alpha}(a)=s(a)$ for any $a \in E$. Then $\mathcal{S}(E)$ is a compact convex Hausdorff space, and due to the Krein-Mil'man Theorem, see [Goo, Thm 5.17], every state on $E$ is a weak limit of a net of convex combinations of extremal states. 


\section{Relatively Bounded Homomorphisms}

A poset $X$ is said to be directed if given $x, y \in X$, there is an element $z \in X$ such that $x \leq z$ and $y \leq z$. It is easy to show that a po-group $G$ is directed iff every element $g \in G$ is a difference of two elements from $G^{+}$, i.e. $g=g_{1}-g_{2}=-g_{1}^{\prime}+g_{2}^{\prime}$, where $g_{1}, g_{2}, g_{1}^{\prime}, g_{2}^{\prime} \in G^{+}$.

Let $(G ;+, 0, \leq)$ be a po-group. A subgroup $H$ of $G$ is said to be convex if from $x \leq y \leq z$, where $x, z \in H$ and $y \in G$, we have $y \in H$. An $o$-ideal is any directed convex subgroup of $G$.

According to DvVe1, DvVe2, we introduce different types of the Riesz Decomposition Properties for po-groups.

Let $(G ;+, 0, \leq)$ be a directed po-group.

(a) For $a, b \in G^{+}$, we write $a \operatorname{com} b$ to mean that for all $a_{1} \leq a$ and $b_{1} \leq b, a_{1}$ and $b_{1}$ commute, where $a_{1}, b_{1} \in G^{+}$.

(b) We say that $G$ fulfils the Riesz Interpolation Property, (RIP) for short, if for any $a_{1}, a_{2}, b_{1}, b_{2} \geq 0$ such that $a_{1}, a_{2} \leq b_{1}, b_{2}$, there is a $c \in G$ such that $a_{1}, a_{2} \leq c \leq b_{1}, b_{2}$.

(c) We say that $G$ fulfils the weak Riesz Decomposition Property, $\left(\mathrm{RDP}_{0}\right)$ for short, if for any $a, b_{1}, b_{2} \geq 0$ such that $a \leq b_{1}+b_{2}$, there are $d_{1}, d_{2} \in G$ such that $0 \leq d_{1} \leq b_{1}, 0 \leq d_{2} \leq b_{2}$ and $a=d_{1}+d_{2}$.

(d) We say that $G$ fulfils the Riesz Decomposition Property, (RDP) for short, if for any $a_{1}, a_{2}, b_{1}, b_{2} \geq 0$ such that $a_{1}+a_{2}=b_{1}+b_{2}$, there are $d_{1}, d_{2}, d_{3}, d_{4} \geq$ 0 such that $d_{1}+d_{2}=a_{1}, d_{3}+d_{4}=a_{2}, d_{1}+d_{3}=b_{1}, d_{2}+d_{4}=b_{2}$.

(e) We say that $G$ fulfils the commutational Riesz Decomposition Property, $\left(\mathrm{RDP}_{1}\right)$ for short, if for any $a_{1}, a_{2}, b_{1}, b_{2} \geq 0$ such that $a_{1}+a_{2}=b_{1}+b_{2}$, there are $d_{1}, d_{2}, d_{3}, d_{4} \geq 0$ such that (i) $d_{1}+d_{2}=a_{1}, d_{3}+d_{4}=a_{2}$, $d_{1}+d_{3}=b_{1}, d_{2}+d_{4}=b_{2}$, and (ii) $d_{2} \operatorname{com} d_{3}$.

(f) We say that $G$ fulfils the strong Riesz Decomposition Property, $\left(\mathrm{RDP}_{2}\right)$ for short, if for any $a_{1}, a_{2}, b_{1}, b_{2} \geq 0$ such that $a_{1}+a_{2}=b_{1}+b_{2}$, there are $d_{1}, d_{2}, d_{3}, d_{4} \geq 0$ such that (i) $d_{1}+d_{2}=a_{1}, d_{3}+d_{4}=a_{2}, d_{1}+d_{3}=b_{1}$, $d_{2}+d_{4}=b_{2}$, and (ii) $d_{2} \wedge d_{3}=0$.

Then

$$
\left(\mathrm{RDP}_{2}\right) \Rightarrow\left(\mathrm{RDP}_{1}\right) \Rightarrow(\mathrm{RDP}) \Rightarrow\left(\mathrm{RDP}_{0}\right) \Leftrightarrow(\mathrm{RIP})
$$

and if $G$ is Abelian, then $\left(\mathrm{RDP}_{0}\right) \Leftrightarrow\left(\mathrm{RDP}_{1}\right)$; if $G$ is not Abelian, the converse implications do not hold, in general, DvVe1, DvVe2. In addition, $\left(\mathrm{RDP}_{2}\right)$ holds in $G$ iff $G$ is an $\ell$-group.

Let $G$ and $H$ be po-groups. A mapping $d: G^{+} \rightarrow H$ is said to be subadditive provided $d(0)=0$ and $d(x+y) \leq d(x)+d(y)$ for all $x, y \in G^{+}$.

The following result is proved in [Goo, Lem 2.24] for Abelian po-groups. An analogous proof can be used also for po-groups with (RIP) that are not necessarily Abelian. Also the rest of this section follows basic ideas of [Goo, pp. 38-42]. Because we are interesting in general po-groups that are not studied in [Goo, and (RIP) is not equivalent with (RDP) for non-Abelian po-groups, we will present all our proofs in full details for our not necessarily commutative po-groups with (RDP).

Proposition 3.1. Let $G$ be a directed po-group with (RDP), and let $H$ be a Dedekind complete $\ell$-group, and let $d: G^{+} \rightarrow H$ be a subadditive mapping. For 
all $x \in G^{+}$, assume that the set

$$
D(x):=\left\{d\left(x_{1}\right)+\cdots+d\left(x_{n}\right): x=x_{1}+\cdots+x_{n}, x_{1}, \ldots, x_{n} \in G^{+}\right\}
$$

is bounded above in $H$. Then there is a group homomorphism $f: G \rightarrow H$ such that $f(x)=\bigvee D(x)$ for all $x \in G^{+}$.

Proof. Since $H$ is a Dedekind complete $\ell$-group, due to [Fuc Cor V.20], $H$ is Abelian.

Therefore, $f(x):=\bigvee D(x)$ is a well-defined mapping for all $x \in G^{+}$. It is clear that $f(0)=0$ and we are now going to show that $f$ is additive on $G^{+}$.

Let $x, y \in G^{+}$be given. For all decompositions

$$
x=x_{1}+\cdots+x_{n} \text { and } y=y_{1}+\cdots+y_{k}
$$

with all $x_{i}, y_{j} \in G^{+}$, we have $x+y=x_{1}+\cdots+x_{n}+y_{1}+\cdots+y_{k}$, that yields

$$
\sum d\left(x_{i}\right)+\sum d\left(y_{j}\right) \leq f(x+y) .
$$

Therefore, $u+v \leq f(x+y)$ for all $u \in D(x)$ and $b \in D(y)$. Since $H$ is Dedekind complete, $\bigvee$ is distributive with respect to + :

$$
\begin{aligned}
f(x)+f(y) & =(\bigvee D(x))+f(y)=\bigvee_{u \in D(x)}(u+f(y)) \\
& =\bigvee_{u \in D(x)}(u+(\bigvee D(y)))=\bigvee_{u \in D(x)} \bigvee_{v \in D(y)}(u+v) \\
& \leq f(x+y) .
\end{aligned}
$$

Conversely, let $x+y=z_{1}+\cdots+z_{n}$, where each $z_{i} \in G^{+}$. Then (RDP) implies that there are elements $x_{1}, \ldots, x_{n}, y_{1}, \ldots, y_{n} \in G^{+}$such that $x=x_{1}+\cdots+x_{n}$, $y=y_{1}+\cdots+y_{n}$ and $z_{i}=x_{i}+y_{i}$ for $i=1, \ldots, n$. This yields

$$
\sum_{i} d\left(z_{i}\right) \leq \sum_{i}\left(d\left(x_{i}\right)+d\left(y_{i}\right)\right)=\left(\sum_{i} d\left(x_{i}\right)\right)+\left(\sum_{i} d\left(y_{i}\right)\right) \leq f(x)+f(y),
$$

and therefore, $f(x+y) \leq f(x)+f(y)$ and finally, $f(x+y)=f(x)+f(y)$ for all $x, y \in G^{+}$.

Since $G$ is directed and $H$ commutative, we can extend $f$ also to the whole $G$ as follows. The positive cone $G^{+}$is a normal cone that generates $G$, so that every element $x \in G$ can be expressed by $x=x_{1}-x_{2}=-x_{1}^{\prime}+x_{2}^{\prime}$ for some $x_{1}, x_{2}, x_{1}^{\prime}, x_{2}^{\prime} \in$ $G^{+}$. Then $x_{1}^{\prime}+x_{1}=x_{2}^{\prime}+x_{2}$ so that $f\left(x_{1}\right)-f\left(x_{2}\right)=-f\left(x_{1}^{\prime}\right)+f\left(x_{2}\right)$. This implies that if also $x=y_{1}-y_{2}$ for some $y_{1}, y_{2} \in G^{+}$, then $f\left(x_{1}\right)-f\left(x_{2}\right)=f\left(y_{1}\right)-f\left(y_{2}\right)$, so that $f$ can be extended by $f(x):=f\left(x_{1}\right)-f\left(x_{2}\right)$ whenever $x=x_{1}-x_{2}$ for $x_{1}, x_{2} \in G^{+}$. In a similar way, we have also $f(x)=-f\left(z_{1}\right)+f\left(z_{2}\right)=f\left(z_{2}\right)-f\left(z_{1}\right)$ whenever $x=-z_{1}+z_{2}$ for some $z_{1}, z_{2} \in G^{+}$.

Let $a \in G^{+}$and $b \in G$ be arbitrary. Then there is an element $a^{\prime} \in G^{+}$such that $a+b=b+a^{\prime}$, so that $a^{\prime}=-b+a+b$. Let $b=b_{1}-b_{2}=-b_{1}+b_{2}$, where $b_{1}, b_{2}, b_{1}^{\prime}, b_{2}^{\prime} \in G^{+}$. Hence,

$$
\begin{gathered}
a+b_{1}-b_{2}=-b_{1}^{\prime}+b_{2}^{\prime}+a^{\prime} \\
b_{1}^{\prime}+a+b_{1}=b_{2}^{\prime}+a^{\prime}+b_{2} \\
f\left(b_{1}^{\prime}\right)+f(a)+f\left(b_{1}\right)=f\left(b_{2}^{\prime}\right)+f\left(a^{\prime}\right)+f\left(b_{2}\right) \\
f(a)+f(b)=f(b)+f\left(a^{\prime}\right)
\end{gathered}
$$


and the commutativity of $H$ entails $f(a)=f\left(a^{\prime}\right)$.

Similarly, there is a unique element $a^{\prime \prime} \in G^{+}$such that $a^{\prime \prime}+b=b+a$ and therefore, $f\left(a^{\prime \prime}\right)=f(a)$.

Now we show that $f(x+y)=f(x)+f(y)$ for all $x, y \in G$. Then $x+y=u-v$, $x=-x_{1}+x_{2}$ and $y=y_{1}-y_{2}$ for some $x_{1}, x_{2}, y_{1}, y_{2}, u, v \in G^{+}$. Then $u-v=$ $-x_{1}+x_{2}+y_{1}-y_{2}$. There are unique elements $x_{2}^{\prime}, y_{1}^{\prime} \in G^{+}$such that

$$
\begin{gathered}
u-v=-x_{1}+x_{2}+y_{1}-y_{2} \\
u-v=-x_{2}-y_{1}+x_{2}^{\prime}+y_{1}^{\prime} \\
y_{2}+x_{1}+u=x_{2}^{\prime}+y_{1}^{\prime}+v \\
f\left(y_{2}\right)+f\left(x_{1}\right)+f(u)=f\left(x_{2}^{\prime}\right)+f\left(y_{1}^{\prime}\right)+f(v) \\
f\left(y_{2}\right)+f\left(x_{1}\right)+f(u)=f\left(x_{2}\right)+f\left(y_{1}\right)+f(v) \\
f(u)-f(v)=-f\left(x_{1}\right)+f\left(x_{2}\right)+f\left(y_{1}\right)-f\left(y_{2}\right) \\
f(x+y)=f(x)+f(y) .
\end{gathered}
$$

This implies that $f$ is a group homomorphism.

Let $X$ and $Y$ be two posets. A mapping $f: X \rightarrow Y$ is said to be relatively bounded provided that given any subset $W$ of $X$ which is bounded (above and below) in $X$, the set $f(W)$ is bounded in $Y$.

We recall that a group homomorphism $f$ from one po-group, $G$, into another one, $H$, is positive if $f\left(G^{+}\right) \subseteq H^{+}$.

Proposition 3.2. Let $G$ be a directed po-group with (RDP), let $H$ be a Dedekind complete $\ell$-group, and let $f: G \rightarrow H$ be a group homomorphism. Then $f$ is relatively bounded if and only if $f=g-h$ for some positive homomorphisms $g, h: G \rightarrow H$.

Proof. Again, $H$ is an Abelian $\ell$-group. Assume that $f=g-h$ for some two positive group homomorphisms $g, h: G \rightarrow H$. If $W \subseteq[a, b]$ in $G$, then $g(W) \subseteq[g(a), g(b)]$ and $h(W) \subseteq[h(a), h(b)]$. Then $g(a)-h(b) \leq g(b)-h(a)$ and $f(W) \subseteq[g(a)-$ $h(b), g(b)-h(a)]$ that proves that $f$ is relatively bounded.

Conversely, let $f$ be relatively bounded. If we set $d(x):=f(x) \vee 0$ for all $x \in G^{+}$, then $d(0)=0$. For all $x, y \in G^{+}$, we have

$$
d(x+y)=(f(x)+f(y)) \vee 0 \leq(f(x) \vee 0)+(f(y) \vee 0)=d(x)+d(y),
$$

so that $d$ is subadditive.

Let us define $D(x)$ by (3.1) for each $x \in G^{+}$. We assert that $D(x)$ is bounded above in $H$. By the assumption, there are elements $a, b \in H$ such that $f([0, x]) \subseteq$ $[a, b]$. Fix a decomposition $x=x_{1}+\cdots+x_{n}$ with $x_{i} \in G^{+}$for each $i=1, \ldots, n$. By [Goo, Lem 1.21], we have

$$
\sum_{i=1}^{n} d\left(x_{i}\right)=\sum_{i=1}^{n}\left(f\left(x_{i}\right) \vee 0\right)=\left(\bigvee_{A \in 2^{n}}\left(\sum_{i \in A} f\left(x_{i}\right)\right)\right) \vee 0 .
$$

For all $A \in 2^{n}$, we have

$$
0 \leq \sum_{i \in A} x_{i} \leq x, \text { and } \sum_{i \in A} f\left(x_{i}\right)=f\left(\sum_{i \in A} x_{i}\right) \leq b .
$$

Hence, $d\left(x_{1}\right)+\cdots+d\left(x_{n}\right) \leq b \vee 0$, and consequently, $b \vee 0$ is an upper bound for $D(x)$ that proves the assertion. 
By Proposition 3.1. there exists a group homomorphism $g: G \rightarrow H$ such that $g(x)=\bigvee D(x)$ for all $x \in G^{+}$. Since $g(x) \geq d(x) \geq 0, g(x)$ is a positive homomorphism, and $g(x) \geq d(x) \geq f(x)$ for all $x \in G^{+}$. Hence, $h=g-f$ is a positive homomorphism, too.

Let $G$ be a directed po-group and $H$ an Abelian po-group. The set, $\operatorname{Hom}(G, H)$, of all group homomorphisms from $G$ into $H$ is an Abelian group. Given $f, g \in$ $\operatorname{Hom}(G, H)$, we define $f \leq^{+} g$ whenever $g-f$ is a positive group homomorphism. Then $\leq^{+}$is a partial order and $\operatorname{Hom}(G, H)$ is an Abelian po-group with respect to this partial order. Indeed, it is easy to see that $\leq^{+}$is a preorder. Assume now $(f-g)(x) \geq 0$ and $(g-f)(x)$ for all $x \in G^{+}$, and thus $f(x)=g(x)$ for all $x \in G^{+}$. The group $G$ is directed and $G^{+}$is a normal cone of $G$ that generates $G$ as a group. If $x=x_{1}-x_{2}$ with $x_{1}, x_{2} \in G^{+}$, then $f(x)=f\left(x_{1}\right)-f\left(x_{2}\right)=g\left(x_{1}\right)-g\left(x_{2}\right)=g(x)$, we see that $f(x)=g(x)$ for all $x \in G$.

It is now clear that the positive cone of $\operatorname{Hom}(G, H)$ consists of all positive homomorphisms from $G$ into $H$. It is non-void because the zero homomorphism from $G$ into $H$ belongs to it.

Proposition 3.3. Let $G$ be a directed po-group with (RDP), let $H$ be a Dedekind complete $\ell$-group, and let $B(G, H)$ be the set of all relatively bounded group homomorphisms from $G$ to $H$. Then $B$ is a nonempty o-ideal of $\operatorname{Hom}(G, H)$.

Proof. Because the zero homomorphism from $G$ to $H$ is a relatively bounded group homomorphism, $B(G, H)$ is non-void, and according to Proposition $3.2 B(G, H)$ equals the subgroup of $\operatorname{Hom}(G, H)$ generated by the positive homomorphisms. Therefore, $B(G, H)$ is a directed subgroup of $\operatorname{Hom}(G, H)$.

Given $f \in \operatorname{Hom}(G, H)$ and $g \in B(G, H)$ such that $0 \leq^{+} f \leq^{+} g$, write $g=g_{1}-g_{2}$ for some positive homomorphisms $g_{1}, g_{2} \in \operatorname{Hom}(G, H)$. Since $f \leq^{+} g \leq^{+} g_{1}$, we have $f=g_{1}-\left(g_{1}-f\right)$ with $g_{1}$ and $g_{1}-f$ positive homomorphisms, and hence, $f \in B(G, H)$. This proves that $B(G, H)$ is an o-ideal.

Theorem 3.4. Let $G$ be a directed po-group with (RDP) and let $H$ be a Dedekind complete $\ell$-group.

(a) The group $B(G, H)$ of all relatively bounded group homomorphisms from $G$ to $H$ is a Dedekind complete $\ell$-group.

(b) If $\left\{f_{i}\right\}_{i \in I}$ is a nonempty system of $B(G, H)$ that is bounded above, and if $d(x)=\bigvee_{i} f_{i}(x)$ for all $x \in G^{+}$, then

$$
\left(\bigvee_{i} f_{i}\right)(x)=\bigvee\left\{d\left(x_{1}\right)+\cdots+d\left(x_{n}\right): x=x_{1}+\cdots+x_{n}, x_{1}, \ldots, x_{n} \in G^{+}\right\}
$$

for all $x \in G^{+}$.

(c) If $\left\{f_{i}\right\}_{i \in I}$ is a nonempty system of $B(G, H)$ that is bounded below, and if $e(x)=\bigwedge_{i} f_{i}(x)$ for all $x \in G^{+}$, then

$$
\left(\bigwedge_{i} f_{i}\right)(x)=\bigwedge\left\{e\left(x_{1}\right)+\cdots+e\left(x_{n}\right): x=x_{1}+\cdots+x_{n}, x_{1}, \ldots, x_{n} \in G^{+}\right\}
$$

for all $x \in G^{+}$.

Proof. Let $g \in B(G, H)$ be an upper bound for $\left\{f_{i}\right\}$. For any $x \in G^{+}$, we have $f_{i}(x) \leq g(x)$, so that the mapping $d(x)=\bigvee_{i} f_{i}(x)$ defined on $G^{+}$is a a subadditive 
mapping. For any $x \in G^{+}$and any decomposition $x=x_{1}+\cdots+x_{n}$ with all $x_{i} \in G^{+}$, we conclude $d\left(x_{1}\right)+\cdots+d\left(x_{n}\right) \leq g\left(x_{1}\right)+\cdots+g\left(x_{n}\right)=g(x)$. Hence, $g(x)$ is an upper set for $D(x)$ defined by (3.1).

Proposition 3.1 entails there is a group homomorphism $f: G \rightarrow H$ such that $f(x)=\bigvee D(x)$. For every $x \in G^{+}$and every $f_{i}$ we have $f_{i}(x) \leq d(x) \leq f(x)$ that gives $f_{i} \leq^{+} f$. The mappings $f-f_{i}$ are positive homomorphisms belonging bo $B(G, H)$ that gives $f \in B(G, H)$. If $h \in B(G, H)$ such that $f_{i} \leq^{+} h$ for any $i \in I$, then $d(x) \leq h(x)$ for any $x \in G^{+}$. As above, we can show that $h(x)$ is also an upper bound for $D(x)$, whence $f(x) \leq h(x)$ for any $x \in G^{+}$that gives $f \leq^{+} h$. In other words, we have proved that $f$ is the supremum of $\left\{f_{i}\right\}_{i \in I}$, and its form is given by (b).

Now if we apply the order anti-automorphism $z \mapsto-z$ in $H$, we see that infima exist in $B(G, H)$ for any bounded below system $\left\{f_{i}\right\}_{i \in I}$, and their form is given by (c).

By Proposition 3.2, $B(G, H)$ is directed, combining (b) and (c), we see that $B(G, H)$ is a Dedekind complete $\ell$-group.

If $H=\mathbb{R}$, Theorem 3.4 can be reformulated as follows.

Theorem 3.5. If $G$ is a directed po-group with (RDP), then the group $B(G, \mathbb{R})$ of all relatively bounded group homomorphisms from $G$ to $\mathbb{R}$ is a Dedekind complete lattice ordered real vector space. Given $f_{1}, \ldots, f_{n} \in B(G, \mathbb{R})$,

$$
\begin{aligned}
& \left(\bigvee_{i=1}^{n} f_{i}\right)(x)=\sup \left\{f_{1}\left(x_{1}\right)+\cdots+f_{n}\left(x_{n}\right): x=x_{1}+\cdots+x_{n}, x_{1}, \ldots, x_{n} \in G^{+}\right\} \\
& \left(\bigwedge_{i=1}^{n} f_{i}\right)(x)=\inf \left\{f_{1}\left(x_{1}\right)+\cdots+f_{n}\left(x_{n}\right): x=x_{1}+\cdots+x_{n}, x_{1}, \ldots, x_{n} \in G^{+}\right\}
\end{aligned}
$$

for all $x \in G^{+}$.

Proof. Due to Theorem 3.4 $B(G, \mathbb{R})$ is a Dedekind complete $\ell$-group. It is evident that it is a Riesz space, i.e., a lattice ordered real vector space.

Take $f_{1}, \ldots, f_{n} \in B(G, \mathbb{R})$ and let $f=f_{1} \vee \cdots \vee f_{n}$. For any $x \in G^{+}$and $x=x_{1}+\cdots+x_{n}$ with $x_{1}, \ldots, x_{n} \in G^{+}$, we have $f_{1}\left(x_{1}\right)+\cdots+f_{n}\left(x_{n}\right) \leq f\left(x_{1}\right)+$ $\cdots+f\left(x_{n}\right)=f(x)$. Due to Theorem 3.4, given an arbitrary real number $\epsilon>0$, there is a decomposition $x=y_{1}+\cdots+y_{k}$ with $y_{1}, \ldots, y_{k} \in G^{+}$such that

$$
\sum_{j=1}^{k} \max \left\{f_{1}\left(y_{j}\right), \ldots, f_{n}\left(y_{j}\right)\right\}>f(x)-\epsilon .
$$

We note that if $a \in G^{+}$and $b \in G$, the elements $a^{\prime}, a^{\prime \prime} \in G^{+}$such that $a+b=$ $b+a^{\prime}$ and $b+a=a^{\prime \prime}+b$ are said to be (right and left) conjugates of $a$ by $b$. Since $\mathbb{R}$ is Abelian, for any $h \in B(G, \mathbb{R}), h\left(a^{\prime}\right)=h(a)=h\left(a^{\prime \prime}\right)$.

If $k<n$, we can add the zero element to the decomposition, if necessary, so that without loss of generality, we can assume that $k \geq n$.

We decompose the set $\{1, \ldots, k\}$ into mutually disjoint sets $J(1), \ldots, J(n)$ such that

$$
J(i):=\left\{j \in\{1, \ldots, k\}: \max \left\{f_{1}\left(y_{j}\right), \ldots, f_{n}\left(y_{j}\right)\right\}=f_{i}\left(y_{j}\right)\right\} .
$$


Assume $J(1)=\left\{j_{t_{1}}, \ldots, j_{n_{1}}\right\}$. Since $G^{+}$is a normal cone of $G, x$ can be expressed in the form $x=x_{j_{t_{1}}}+\cdots+x_{j_{n_{1}}}+x_{j}^{\prime}+\cdots+x_{k}^{\prime}$, where $x_{j}^{\prime}, \ldots, x_{k}^{\prime} \in G^{+}$are conjugates of $x_{j}, \ldots, x_{k}$.

Let $x_{1}:=x_{j_{t_{1}}}+\cdots+x_{j_{n_{1}}}$.

In a similar way, let $J(2)=\left\{j_{t_{2}}, \ldots, j_{n_{2}}\right\}$ and let $x_{2}=y_{j_{t_{2}}}+\cdots+y_{j_{n_{2}}}$. Again, we can express $x$ in the form $x=x_{1}+x_{2}+y_{s}^{\prime \prime}+\cdots+y_{k}^{\prime \prime}$, where $y_{t}^{\prime \prime \prime}$ s are appropriate conjugates of $y_{s}^{\prime}, \ldots, y_{k}^{\prime}$. Processing in this way for each $J(i)=\left\{j_{t_{i}}, \ldots, j_{n_{i}}\right\}$, we define the element $x_{i}=c_{t_{j_{t_{i}}}}+\cdots+c_{t_{j_{n_{i}}}}$, where $c_{t_{j_{s}}}$ is an appropriate conjugate of the element $y_{t_{j_{s}}}$. Then $x=x_{1}+\cdots+x_{n}$, and

$$
\sum_{i=1}^{n} f_{i}\left(x_{i}\right)=\sum_{i=1}^{n} \sum_{j \in J(i)} f_{i}\left(y_{j}\right)=\sum_{i=1}^{k} \max \left\{f_{1}\left(y_{j}\right), \ldots, f_{n}\left(y_{j}\right)\right\}>f(x)-\epsilon .
$$

This implies $f(x)$ equals the given supremum.

The formula for $\left(f_{1} \wedge \cdots \wedge f_{n}\right)(x)$ can be obtained applying the order antiautomorphism $z \mapsto-z$ holding in $\mathbb{R}$.

\section{State Spaces of Pseudo Effect Algebras and Simplices}

Simplices are important mathematical tools that can be used also for analysis of the state space of a pseudo effect algebra. In particular, we show that, for any pseudo effect algebra $E$ with $(\mathrm{RDP})_{1}$, the state space of $E$ is either an empty set or it is a nonempty simplex. This result generalizes analogous result holding for effect algebras, see [Dvu, Thm 5.1].

Now we present some elements of simplices. For a good source about convex sets, see the monographs Alf, Phe, Goo, AlSc.

Let $K_{1}, K_{2}$ be two convex sets. A mapping $f: K_{1} \rightarrow K_{2}$ is said to be affine if it preserves all convex combinations, and if $f$ is also injective and surjective such that also $f^{-1}$ is affine, $f$ is an affine isomorphism and $K_{1}$ and $K_{2}$ are affinely isomorphic.

We recall that a convex cone in a real linear space $V$ is any subset $C$ of $V$ such that (i) $0 \in C$, (ii) if $x_{1}, x_{2} \in C$, then $\alpha_{1} x_{1}+\alpha_{2} x_{2} \in C$ for any $\alpha_{1}, \alpha_{2} \in \mathbb{R}^{+}$. A strict cone is any convex cone $C$ such that $C \cap-C=\{0\}$, where $-C=\{-x: x \in C\}$. A base for a convex cone $C$ is any convex subset $K$ of $C$ such that every non-zero element $y \in C$ may be uniquely expressed in the form $y=\alpha x$ for some $\alpha \in \mathbb{R}^{+}$and some $x \in K$.

We recall that in view of [Goo, Prop 10.2], if $K$ is a non-void convex subset of $V$, and if we set

$$
C=\left\{\alpha x: \alpha \in \mathbb{R}^{+}, x \in K\right\},
$$

then $C$ is a convex cone in $V$, and $K$ is a base for $C$ iff there is a linear functional $f$ on $V$ such that $f(K)=1$ iff $K$ is contained in a hyperplane in $V$ which misses the origin.

Any strict cone $C$ of $V$ defines a partial order $\leq_{C}$ via $x \leq_{C} y$ iff $y-x \in C$. It is clear that $C=\left\{x \in V: 0 \leq_{C} x\right\}$. A lattice cone is any strict convex cone $C$ in $V$ such that $C$ is a lattice under $\leq_{C}$.

A simplex in a linear space $V$ is any convex subset $K$ of $V$ that is affinely isomorphic to a base for a lattice cone in some real linear space. A simplex $K$ in 
a locally convex Hausdorff space is said to be (i) Choquet if $K$ is compact, and (ii) Bauer if $K$ and $\partial_{e} K$ are compact, where $\partial_{e} K$ is the set of extreme points of $K$.

For example, for the important quantum mechanical example, if $H$ is a separable complex Hilbert space, $\mathcal{S}(\mathcal{E}(H))$ is not a simplex due to [AlSc, Thm 4.4] or [BrRo, Ex 4.2.6], where $\mathcal{E}(H)$ is the system of all Hermitian operators on a Hilbert space that are between the zero operator and the identity operator. On the other hand, the state space of a commutative $\mathrm{C}^{*}$-algebra and the trace space of a general $\mathrm{C}^{*}$ algebra are Choquet simplices, see [AlSc, p. 7] or [BrRo, Ex 4.2.6].

Let $(G, u)$ be a unital po-group with strong unit. A state on $(G, u)$ we understand any positive homomorphism $s: G \rightarrow \mathbb{R}$ that is normalized, i.e. $s(u)=1$. Let $\mathcal{S}(G, u)$ be the set of all states on $(G, u)$. If $(G, u)$ is an Abelian po-group, due to Goo, Cor 4.3], $\mathcal{S}(G, u)$ is always nonempty, whenever $G \neq\{0\}$. This is not true, in general, for non-Abelian unital po-groups, even not for unital $\ell$-groups, see [Dvu, Cor 4.7]. On the other hand, if $(G, u)$ is a linearly ordered, then $\mathcal{S}(G, u)$ is a singleton, Dvu, Thm 5.6]. It is possible to show [Dvu, Prop 4.3, 4.6] that for a unital $\ell$-group $(G, u)$, a state $s$ is extremal iff $\operatorname{Ker}(s):=\{g \in G: s(|g|)=0\}$ is a maximal $\ell$-ideal (= lattice ordered o-ideal) that is normal. Therefore, $(G, u)$ is non-void iff the unital $\ell$-group $(G, u)$ has at least one maximal $\ell$-ideal that is also normal.

In a similar way as for pseudo effect algebras, we can define an extremal state, and let $\partial_{e} \mathcal{S}(G, u)$ be the set of extremal states on $(G, u)$. We say that a net of states, $\left\{s_{\alpha}\right\}$, on $(G, u)$ converges weakly to a state, $s$, if $\lim _{\alpha} s_{\alpha}(g)=s(g)$ for any $g \in G$. Then $\mathcal{S}(G, u)$ is a compact convex Hausdorff space, and due to the KreinMil'man Theorem, see [Goo, Thm 5.17], every state on $(G, u)$ is a weak limit of a net of convex combinations of extremal states.

Proposition 4.1. Let $E=\Gamma(G, u)$, where $(G, u)$ is a unital po-group satisfying (RDP). Then the restriction of every state $s$ on $E$ gives a state on $E$, and conversely, every state on $E$ can be uniquely extended to a state on $(G, u)$. Moreover, the state spaces $\mathcal{S}(G, u)$ and $\mathcal{S}(E)$ are affinelly homeomorphic.

Proof. It is evident that the restriction of any state on $(G, u)$ is a state on $E$. Conversely, let $s$ be a state on $E$. We extend $s$ onto a state $\hat{s}$ defined on $G^{+}$via $\hat{s}(x)=s\left(x_{1}\right)+\cdots+s\left(x_{n}\right)$ whenever $x=x_{1}+\cdots+x_{n}$, where $x_{1}, \ldots, x_{n} \in E$. We show that $\hat{s}$ is well-defined, indeed, let $x=y_{1}+\cdots+y_{m}$ with $y_{1}, \ldots, y_{m} \in E$. The (RDP) entails that there is a finite system $\left\{c_{i j}: i=1, \ldots, n, j=1, \ldots, m\right\}$ from $G^{+}$such that every $x_{i}=\sum_{j=1}^{m} c_{i j}$. This implies each $c_{i j}$ is from $E$. Check: $\sum_{i=1}^{n} s\left(x_{i}\right)=\sum_{i=1}^{n} \sum_{j=1}^{m} s\left(c_{i j}\right)=\sum_{j=1}^{m} s\left(y_{j}\right)$.

Since $\hat{s}$ is additive on $G^{+}$and $G^{+}$generates $G, \hat{s}$ can be easily extended to a unique state on the whole $G$.

Therefore, the mapping $s \mapsto \hat{s}$ defined on $\mathcal{S}(E)$ is injective, surjective and continuous. If $s \in \partial_{e} \mathcal{S}(E)$, then $\hat{s} \in \partial_{e} \mathcal{S}(G, u)$, and vice versa.

Therefore, the state spaces $\mathcal{S}(E)$ and $\mathcal{S}(G, u)$ are affinelly homeomorphic.

Theorem 4.2. If $(G, u)$ is a unital po-group with $(\mathrm{RDP})$, then either $\mathcal{S}(G, u)$ is empty or it is a nonempty Choquet simplex. In addition, the same is true for $\mathcal{S}(\Gamma(G, u))$.

Proof. Assume that $\mathcal{S}(G, u)$ is nonempty. Then the positive cone $B(G, \mathbb{R})^{+}$of the Abelian Dedekind complete $\ell$-group $B(G, \mathbb{R})$ consists of all positive homomorphisms from $G$ into $\mathbb{R}$, so that $B(G, \mathbb{R})^{+}=\left\{\alpha s: \alpha \in \mathbb{R}^{+}, s \in \mathcal{S}(G, u)\right\}$. The set $\mathcal{S}(G, u)$ 
lies in the hyperplane $\{f \in B(G, \mathbb{R}): f(u)=1\}$ which misses the origin. Therefore, $\mathcal{S}(G, u)$ is a base for $B(G, \mathbb{R})^{+}$, and $\mathcal{S}(G, u)$ is a simplex. Since $\mathcal{S}(G, u)$ is compact, $\mathcal{S}(G, u)$ is a Choquet simplex.

Since $\mathcal{S}(G, u)$ and $\mathcal{S}(\Gamma(G, u))$ are affinely homeomorphic, Proposition 4.1, we conclude that $\mathcal{S}(\Gamma(G, u))$ is also a Choquet simplex.

Theorem 4.3. Let $E$ be a pseudo effect algebra with $(\mathrm{RDP})_{1}$. Then $\mathcal{S}(E)$ is either an empty set or it is a nonempty Choquet simplex.

Proof. Let $E$ be a pseudo effect algebra with $(\mathrm{RDP})_{1}$ and let $\mathcal{S}(E) \neq \emptyset$. Due to the basic representation of pseudo effect algebras with (RDP) $)_{1}$, DvVe2, Thm 5.7], there is a unique (up to isomorphism) unital po-group $(G, u)$ with (RDP) ${ }_{1}$ such that $E \cong \Gamma(G, u)$. Because $(G, u)$ satisfies also (RDP), by Theorem 4.2, $\mathcal{S}(\Gamma(G, u))$ and $\mathcal{S}(E)$ are affinelly isomorphic Choquet simplices.

Theorem 4.4. Let $E$ be a pseudo effect algebra with $(\mathrm{RDP})_{2}$. Then $\mathcal{S}(E)$ is either an empty set or it is a nonempty Bauer simplex.

Proof. By [DvVe2, Thm 5.7], there is a unique unital po-group $(G, u)$ with (RDP) $)_{1}$ such that $E \cong \Gamma(G, u)$. By [DvVe2, Prop. 6.3], $(G, u)$ satisfies even $(\mathrm{RDP})_{2}$.

Assume that $\mathcal{S}(E) \neq \emptyset$. As it was already mentioned at the end of Section 2, $E$ can be converted into a pseudo MV-algebra. Due to [Dvu, Prop 4.7], a state $s$ on a pseudo MV-algebra is extremal iff $s(a \wedge b)=\min \{s(a), s(b)\}$ for all $a, b \in E$. Therefore, $\partial_{e} \mathcal{S}(E)$ is compact, and by Theorem 4.3, $\mathcal{S}(E)$ is a compact simplex. Hence, $\mathcal{S}(E)$ is a nonempty Choquet simplex.

\section{Representation of States by Integrals}

In this main section of the paper, we show that if $s$ is a state on a pseudo effect algebra with $(\mathrm{RDP})_{1}$, then it can be represented as an integral of a continuous affine function through some regular Borel probability measure. It will generalize analogous results from [Dvu2].

We start with some necessary definitions.

Let $K$ be a compact convex subset of a locally convex Hausdorff space. A mapping $f: K \rightarrow \mathbb{R}$ is said to be affine if, for all $x, y \in K$ and any $\lambda \in[0,1]$, we have $f(\lambda x+(1-\lambda) y)=\lambda f(x)+(1-\lambda) f(y)$. Let $\operatorname{Aff}(K)$ be the set of all continuous affine functions on $K$. Then $\operatorname{Aff}(K)$ is a unital po-group with the strong unit 1 which is a subgroup of the po-group $\mathrm{C}(K)$ of all continuous real-valued functions on $K$ (we recall that, for $f, g \in \mathrm{C}(K), f \leq g$ iff $f(x) \leq g(x)$ for any $x \in K$ ), hence it is an Archimedean unital po-group with the strong unit 1 that is even an $\ell$-group.

For example, let $E$ be a pseudo effect algebra such that $\mathcal{S}(E) \neq \emptyset$. Given $a \in E$, let $\hat{a}: \mathcal{S}(E) \rightarrow[0,1]$ such that $\hat{a}(s):=s(a), s \in \mathcal{S}(E)$. Then $\hat{a} \in \operatorname{Aff}(\mathcal{S}(E))$. In a similar way, if $\mathcal{S}(G, u) \neq \emptyset$, for any $g \in(G, u)$, the mapping $\hat{g}: \mathcal{S}(G, u) \rightarrow \mathbb{R}$ defined by $\hat{g}(s):=s(g), s \in \mathcal{S}(G, u)$, is a continuous affine function on $\mathcal{S}(G, u)$.

Let $S=\mathcal{S}(\operatorname{Aff}(K), 1)$. Then the evaluation mapping $\psi: K \rightarrow S$ defined by $\psi(x)(f)=f(x)$ for all $f \in \operatorname{Aff}(K)(x \in K)$ is an affine homeomorphism of $K$ onto $S$, see [Goo, Thm 7.1].

The po-group $\operatorname{Aff}(K)$ is not necessarily neither with (RIP) nor an $\ell$-group. By Goo, Thm 11.4], Aff $(K)$ has (RIP) iff $K$ is a Choquet simplex, and [Goo, Thm 11.21], $\operatorname{Aff}(K)$ is an $\ell$-group iff $K$ is a Bauer simplex. Therefore, due to Theorems 4.2 4.4 we have the following result: 
Theorem 5.1. Let for a unital po-group $(G, u)$, the state space $\mathcal{S}(G, u)$ be nonvoid. If $(G, u)$ has $(\mathrm{RDP})$, then $(\operatorname{Aff}(\mathcal{S}(G, u)), 1)$ is an Abelian unital po-group with (RIP).

If $(G, u)$ has $(\mathrm{RDP})_{2}$, then $(\operatorname{Aff}(\mathcal{S}(G, u)), 1)$ is an Abelian unital po-group with $(\mathrm{RDP})_{2}$.

Let $E$ be a pseudo effect algebra admitting at least one state. If $E$ has $(\mathrm{RDP})_{1}$, then $(\operatorname{Aff}(\mathcal{S}(E)), 1)$ is an Abelian unital po-group with $(\mathrm{RIP})$, if $E$ has $(\mathrm{RDP})_{2}$, then $(\operatorname{Aff}(\mathcal{S}(E)), 1)$ is an Abelian unital $\ell$-group.

If $K$ is a compact Hausdorff topological space, let $\mathcal{B}(K)$ be the Borel $\sigma$-algebra of $K$ generated by all open subsets of $K$. Let $\mathcal{M}_{1}^{+}(K)$ denote the set of all probability measures, that is, all positive regular $\sigma$-additive Borel measures $\mu$ on $\mathcal{B}(K)$. We recall that a Borel measure $\mu$ is called regular if

$$
\inf \{\mu(O): Y \subseteq O, O \text { open }\}=\mu(Y)=\sup \{\mu(C): C \subseteq Y, C \text { closed }\}
$$

for any $Y \in \mathcal{B}(K)$.

For example, if $x \in K$, then the Dirac measure $\delta_{x}$ concentrated at the point $x$ is a regular Borel probability measure on $\mathcal{B}(K)$.

For two measures $\mu$ and $\lambda$ we write

$$
\mu \sim \lambda \quad \text { iff } \quad \int_{K} f \mathrm{~d} \mu=\int_{K} f \mathrm{~d} \lambda, f \in \operatorname{Aff}(K) .
$$

If $\mu$ and $\lambda$ are nonnegative regular Borel measures on a convex compact set $K$, we introduce for them the Choquet ordering defined by

$$
\mu \prec \lambda \quad \text { iff } \quad \int_{K} f \mathrm{~d} \mu \leq \int_{K} f \mathrm{~d} \lambda, f \in \operatorname{Con}(K),
$$

where $\operatorname{Con}(K)$ is the set of all continuous convex functions $f$ on $K$ (that is $f\left(\alpha x_{1}+\right.$ $\left.(1-\alpha) x_{2}\right) \leq \alpha f\left(x_{1}\right)+(1-\alpha) f\left(x_{2}\right)$ for $x_{1}, x_{2} \in K$ and $\left.\alpha \in[0,1]\right)$. Then $\prec$ is a partial order on the cone of nonnegative measures. The fact $\lambda \prec \mu$ and $\mu \prec \lambda$ implies $\lambda=\mu$ follows from the fact that $\operatorname{Con}(K)-\operatorname{Con}(K)$ is dense in $\mathrm{C}(K)$.

Moreover, for any probability measure (= regular Borel probability measure) $\lambda$ there is a maximal probability measure $\mu$ such that $\mu \succ \lambda$, [Phe, Lem 4.1].

The fact $\mu \succ \lambda$ means that $\lambda$ has its support "closer" to the extreme points of $K$ than does $\lambda$.

The following results have been proved in Dvu2 for interval effect algebras. Here we generalize them for pseudo effect algebras with (RDP), $(\mathrm{RDP})_{1}$, and $(\mathrm{RDP})_{2}$, respectively. However, the situation for pseudo effect algebras follows basic ideas of the analogous proofs from Dvu2, we present the proofs here in full generality because it was necessary to take into account a non-commutative character of pseudo effect algebras that was developed in the previous sections.

Theorem 5.2. Let $E=\Gamma(G, u)$ be a pseudo effect algebra such that it admits at least one state, where $(G, u)$ is a unital po-group with (RDP) and let $s$ be a state on $E$. Let $\psi: E \rightarrow \operatorname{Aff}(\mathcal{S}(E))$ be defined by $\psi(a):=\hat{a}, a \in E$, where $\hat{a}$ is a mapping from $\mathcal{S}(E)$ into $[0,1]$ such that $\hat{a}(s):=s(a), s \in \mathcal{S}(E)$. Then there is a unique state $\tilde{s}$ on the unital Abelian po-group $(\operatorname{Aff}(\mathcal{S}(E)), 1)$ such that $\tilde{s}(\hat{a})=s(a)$ for any $a \in E$.

The mapping $s \mapsto \tilde{s}$ defines an affine homeomorphism from the state space $\mathcal{S}(E)$ onto $\mathcal{S}(\Gamma(\operatorname{Aff}(\mathcal{S}(E)), 1))$. 
Proof. By Proposition 4.1, the mapping $\psi$ can be uniquely extended to a po-group homomorphism $\hat{\psi}: G \rightarrow \operatorname{Aff}(\mathcal{S}(E))$ via $\hat{\psi}(g)(s):=s(g), s \in \mathcal{S}(G, u)$. Let $\hat{s}$ be a state on $(G, u)$ that is a unique extension of a state $s$. Now applying the proof of Goo, Prop 7.20], we can show that [Goo, Prop 7.20] holds also for our group $G$ that is not necessarily bounded. Therefore, we have that there is a unique state $\tilde{s}$ on $(\operatorname{Aff}(\mathcal{S}(E)), 1)$ such that $\tilde{s}(\hat{a})=s(a), a \in E$.

The affine homeomorphism $s \mapsto \tilde{s}$ follows from [Goo, Thm 7.1].

Theorem 5.3. Let $(G, u)$ be a unital po-group with (RDP) and let $s$ be a state on the pseudo effect algebra $E=\Gamma(G, u)$. Then there is a unique maximal regular Borel probability measure $\mu_{s} \sim \delta_{s}$ on $\mathcal{B}(\mathcal{S}(E))$ such that

$$
s(a)=\int_{\mathcal{S}(E)} \hat{a}(x) \mathrm{d} \mu_{s}(x), \quad a \in E .
$$

Proof. Due to Theorem 4.2, $\mathcal{S}(E)$ is a Choquet simplex. By Theorem 5.2 , there is a unique state $\tilde{s}$ on $(\operatorname{Aff}(\mathcal{S}(E)), 1)$ such that $\tilde{s}(\hat{a})=s(a), a \in A$.

Applying the Choquet-Meyer Theorem, [Phe, Thm p. 66], we have

$$
f(s)=\int_{\mathcal{S}(E)} f(x) \mathrm{d} \mu_{s}, \quad f \in \operatorname{Aff}(\mathcal{S}(E)) .
$$

Since $\hat{a} \in \operatorname{Aff}(\mathcal{S}(E))$ for any $a \in E$, we have the representation given by (5.1).

Theorem 5.4. Let $E$ be a pseudo effect algebra satisfying $\left(\mathrm{RDP}_{1}\right)$ and let $s$ be a state on $E$. Then there is a unique maximal regular Borel probability measure $\mu_{s} \sim \delta_{s}$ on $\mathcal{B}(\mathcal{S}(E))$ such that

$$
s(a)=\int_{\mathcal{S}(E)} \hat{a}(x) \mathrm{d} \mu_{s}(x), \quad a \in E .
$$

Proof. By Goo, Thm 5.7], there is a unital po-group $(G, u)$ with $(\mathrm{RDP})_{1}$ such that $E \cong \Gamma(G, u)$. The desired result follows now from Theorem 5.3

Theorem 5.5. Let $E$ be a pseudo effect algebra with $(\mathrm{RDP})_{2}$ and let $s$ be a state on $E$. Then there is a unique regular Borel probability measure, $\mu_{s}$, on $\mathcal{B}(\mathcal{S}(E))$ such that $\mu_{s}\left(\partial_{e} \mathcal{S}(E)\right)=1$ and

$$
s(a)=\int_{\partial_{e} \mathcal{S}(E)} \hat{a}(x) \mathrm{d} \mu_{s}(x), \quad a \in E .
$$

Proof. Due to Theorem 5.3, we have a unique regular Borel probability measure $\mu_{s} \sim \delta_{s}$ such that (5.1) holds. The characterization of Bauer simplices, Alf, Thm II.4.1], says that then $\mu_{s}$ is a unique regular Borel probability measure $\mu_{s}$ on such that (5.1) holds and $\mu_{s}\left(\partial_{e} \mathcal{S}(E)\right)=1$. Hence, (5.2) holds.

Corollary 5.6. Let $s$ be a state on a pseudo $M V$-algebra $E$. Then there is a unique regular Borel probability measure, $\mu_{s}$, on $\mathcal{B}(\mathcal{S}(E))$ such that $\mu_{s}\left(\partial_{e} \mathcal{S}(E)\right)=1$ and

$$
s(a)=\int_{\partial_{e} \mathcal{S}(E)} \hat{a}(x) \mathrm{d} \mu_{s}(x), \quad a \in E .
$$


Proof. From [DvVe2, Thm 8.8], we have that the pseudo MV-algebra $E$ can be converted into a pseudo effect algebra with $(\mathrm{RDP})_{2}$. Since the notions of states on pseudo MV-algebras and on pseudo effect algebras coincide, the corollary follows from Theorem 5.5

Corollary 5.6 generalizes the analogous statements proved in [Kro, $\mathrm{Pan}]$ for MValgebras.

We endow the set of regular Borel probability measures with the weak ${ }^{*}$ topology, i.e., a net $\left\{\mu_{\alpha}\right\}$ converges to an element $\mu$ iff $\int_{K} f \mathrm{~d} \mu_{\alpha} \rightarrow \int_{K} f \mathrm{~d} \mu$ for all $f \in \mathrm{C}(K)$.

Any convex subset $F$ of a convex set $K$ is a face if $x=\lambda x_{1}+(1-\lambda) x_{2} \in F$, $0<\lambda<1$, entail $x_{1}, x_{2} \in F$.

Corollary 5.7. Let $E$ be a pseudo effect algebra with $(\mathrm{RDP})_{2}$ and let $\mathcal{S}(E)$ be nonempty. Let $F$ be the set of regular Borel probability measures $\mu \in \mathcal{M}_{1}^{+}(\mathcal{S}(E))$ such that $\mu\left(\partial_{e} \mathcal{S}(E)\right)=1$ is a closed face. The mapping $s \mapsto \mu_{s}$, where $\mu_{s}$ is a unique regular Borel probability measure satisfying $(5.2)$ and $\mu_{s}\left(\partial_{e} \mathcal{S}(E)\right)=1$, is an affine homeomorphism between $\mathcal{S}(E)$ and $F$ that is endowed with the weak* topology. A state $s$ on $E$ is extremal if and only if $\mu_{s}$ in (5.2) is extremal. In such a case, $\mu_{s}=\delta_{s}$.

Proof. Due to Theorem 5.5 and (5.2), the mapping $s \mapsto \mu_{s}$ is affine and injective. If $\mu$ is a regular Borel probability measure with $\mu\left(\partial_{e} \mathcal{S}(E)\right)=1$, then $\mu$ defines via (5.2) some state, $s$, on $E$. Hence, the mapping is surjective. The continuity follows from [Alf, Thm II.4.1(iii)].

It is clear that $F$ is a face. Since the set $\partial_{e} \mathcal{S}(E)$ is closed, due to Goo, Prop $5.25], F$ is closed.

At any rate, every Dirac measure $\delta_{s}$ also with $\delta_{s}\left(\partial_{e} \mathcal{S}(E)\right)=1$ is always a regular Borel probability measure. Equality (5.2) entails that $s$ has to be extremal. Conversely, if $s$ is extremal, the uniqueness of $\mu_{s}$ yields that $\mu_{s}=\delta_{s}$.

It is worthy to remark a note concerning formula (5.2) that if $\mu$ is any regular Borel measure, the formula (5.1) defines a state, say $s_{\mu}$, on $E$. But if $\mu\left(\partial_{e} \mathcal{S}(E)\right)<1$, then for $s_{\mu}$ there is another unique regular Borel probability measure $\mu_{0}$ such that $\mu_{0}\left(\partial_{e} \mathcal{S}(E)\right)=1$ and it represents $s_{\mu}$ via $(5.2)$.

Corollary 5.8. Let $(G, u)$ be a unital po-group satisfying (RDP) and let s be a state on it. Then there is a unique maximal regular Borel probability measure $\mu_{s} \sim \delta_{s}$ on $\mathcal{B}(\mathcal{S}(G, u))$ such that

$$
s(g)=\int_{\mathcal{S}(G, u)} \hat{g}(x) \mathrm{d} \mu_{s}(x), \quad g \in G .
$$

If, in addition, $(G, u)$ satisfies $(\mathrm{RDP})_{2}$, there is a unique regular Borel probability measure, $\mu_{s}$, on $\mathcal{B}(\mathcal{S}(G, u))$ such that $\mu_{s}\left(\partial_{e} \mathcal{S}(G, u)\right)=1$ and

$$
s(g)=\int_{\partial_{e} \mathcal{S}(G, u)} \hat{g}(x) \mathrm{d} \mu_{s}(x), \quad g \in G .
$$

Proof. Due to Proposition 4.1, the state spaces of $E=\Gamma(G, u)$ and of $\Gamma(G, u)$ are affinely homeomorphic and every state on $E$ can be uniquely extended to a state on $(G, u)$. The statements follow easily from Theorem [5.3. (5.1) and from Theorem 5.5 and (5.2), respectively. Indeed, let $s \in \mathcal{S}(G, u)$. We define a mapping 
$\theta: \mathcal{S}(G, u) \rightarrow \mathcal{S}(E)$ defined by $\theta(s):=s \mid E$. By Proposition 4.1 $\theta$ is an affine homeomorphism.

It is enough to assume $g \in G^{+}$. Then $g=a_{1}+\cdots+a_{n}$ with $a_{1}, \ldots, a_{n} \in E$. Then $s(g)=s\left(a_{1}\right)+\cdots+s\left(a_{n}\right)=\theta(s)\left(a_{1}\right)+\cdots+\theta(s)\left(a_{n}\right)=\hat{a}_{1}(\theta(s))+\cdots+$ $\hat{a}_{n}(\theta(s))=\hat{g}(s)$. Let $\nu_{s}$ be a unique regular Borel measure defined on $\mathcal{B}(\mathcal{S}(E))$ such that $\nu_{s} \sim \delta_{\theta(s)}$ and (5.1) holds. If we set $\mu_{s}:=\nu_{s} \circ \theta$, then $\mu_{s}$ is a unique regular measure on $\mathcal{S}(G, u)$ such that $\mu_{s} \sim \delta_{s}$. Then (5.1) gives

$$
s(g)=\sum_{i=1}^{n} \int_{\mathcal{S}(E)} \hat{a}_{i}(y) \mathrm{d} \nu_{s}(y)=\int_{\theta^{-1}(S(E))} \hat{g}(\theta(x)) \mathrm{d} \nu_{s}(\theta(x))=\int_{\mathcal{S}(G, u)} \hat{g}(x) \mathrm{d} \mu_{s}(x) .
$$

\section{Conclusion}

The states on pseudo effect algebras are analogous of probabilities appearing in quantum measurements. In many situations, an effect algebra or a pseudo effect algebra is an interval in a unital po-group. Their state spaces are convex compact Hausdorff spaces that are sometimes empty. If the state space is nonempty, then some kind of the Riesz Decomposition Property allows us to show that the state space is a Choquet simplex, Theorem 4.2, or even a Bauer simplex, Theorem 4.4.

However, the state space of the crucial example of the Hilbert space quantum mechanics, $\mathcal{E}(H)$, is not a simplex, the state spaces of commutative $\mathrm{C}^{*}$-algebra are simplices.

In Section 5, we have showed that if a state of a pseudo effect algebra is with $(\mathrm{RDP})_{1}$ or with $(\mathrm{RDP})_{2}$, then it can be expressed as an integral of some continuous affine function through a regular Borel probability measure, formulas (5.1) and (5.2), even with some kind of uniqueness.

Formulas (5.1) and (5.2) are interesting also in other point of view: According to de Finetti, a probability measure is only a finitely additive measure, and by Kolmogorov [Kol], a probability measure is assumed to be $\sigma$-additive. The mentioned formulas show that there is a natural coexistence between both approaches.

\section{REFERENCES}

[Alf] E.M. Alfsen, "Compact Convex Sets and Boundary Integrals", Springer-Verlag, Berlin, 1971.

[AlSc] E.M. Alfsen, F.W. Schultz, "State Spaces of Operator Algebras", Birkhäuser, BostonBasel-Berlin, 2001.

[BeFo] M.K. Bennett, D.J. Foulis, Phi-symmetric effect algebras, Found. Phys. 25 (1995), 16991722 .

[BrRo] O. Bratteli, D.W. Robinson, "Operator Algebras and Quantum Statistical Mechanics," Springer-Verlag, New York, Heidelberg, Berlin, 1979.

[Dvu] A. Dvurečenskij, States on pseudo MV-algebras, Studia Logica 68 (2001), 301-327.

[Dvu1] A. Dvurečenskij, Pseudo MV-algebras are intervals in $\ell$-groups, J. Austral. Math. Soc., 72 (2002), 427-445.

[Dvu2] A. Dvurečenskij, Every state on interval effect algebra is integral, J. Math. Phys. 51 (2010), to appear.

[DvPu] A. Dvurečenskij, S. Pulmannová, "New Trends in Quantum Structures", Kluwer Acad. Publ., Dordrecht, Ister Science, Bratislava, 2000.

[DvVe1] A. Dvurečenskij, T. Vetterlein, Pseudoeffect algebras. I. Basic properties, Inter. J. Theor. Phys. 40 (2001), 685-701. 
[DvVe2] A. Dvurečenskij, T. Vetterlein, Pseudoeffect algebras. II. Group representation, Inter. J. Theor. Phys. 40 (2001), 703-726.

[FoBe] D.J. Foulis, M.K. Bennett, Effect algebras and unsharp quantum logics, Found. Phys. 24 (1994), 1325-1346.

[Fuc] L. Fuchs, Partially Ordered Algebraic Systems, Pergamon Press, Oxford, London, NY, Paris, 1963).

[GeIo] G. Georgescu, A. Iorgulescu, Pseudo-MV algebras, Multi. Val. Logic 6 (2001), 95-135.

[Gla] A.M.W. Glass "Partially Ordered Groups", World Scientific, Singapore, 1999.

[Goo] K.R. Goodearl, "Partially Ordered Abelian Groups with Interpolation", Math. Surveys and Monographs No. 20, Amer. Math. Soc., Providence, Rhode Island, 1986.

[Kol] A.N. Kolmogorov, "Grundbegriffe der Wahrscheinlichkeitsrechnung", Julius Springer, Berlin, 1933.

[Kro] T. Kroupa, Every state on semisimple $M V$-algebra is integral. Fuzzy Sets and Systems 157 (2006), 2771-2782.

[Pan] G. Panti, Invariant measures in free MV-algebras, Comm. Algebra 36 (2008), 2849-2861.

[Phe] R.R. Phelps, "Lectures on Choquet's Theorem", Van Nostrand, Princeton, 1965.

[Rav] K. Ravindran, On a structure theory of effect algebras, PhD thesis, Kansas State Univ., Manhattan, Kansas, 1996. 